\title{
Is it time for epigenetics in Osteoarthritis?
}

\section{Francisco J. Blanco and Ignacio Rego-Pérez}

Servicio de Reumatología. Instituto de Investigación Biomédica de A Coruña (INIBIC). Complexo Hospitalario Universitario de A Coruña (CHUAC), Sergas. Universidade da Coruña (UDC). As Xubias, 15006. A Coruña, España.

Running title: Epigenetics and Osteoarthritis

Key Words: osteoarthritis, epigenetics, DNA methylation, mitochondria, phenotypes

Funding. This study was supported by grants from Fondo Investigacion Sanitaria PI 12/0329 with a contribution of funds from FEDER (European Community). Ignacio Rego-Pérez is supported by Contrato Miguel Servet-Fondo Investigacion Sanitaria (CP12/03192).

\section{Address:}

Francisco J. Blanco, MD

Rheumatology Service

INIBIC-Complejo Hospitalario Universitario A Coruña

15006-A Coruña, SPAIN

Phone: 34-981-176399

Fax: 34-981-176398

E-mail: fblagar@sergas.es 


\begin{abstract}
DNA methylation is an epigenetic mechanism of reversible gene regulation which typically results in gene silencing upon hypermethylation in high CpG density regions of promoters (termed $\mathrm{CpG}$ islands), and increased gene expression when methylation occurs in gene bodies. Prior information on OA-associated changes in DNA methylation are limited to important single gene analyses and two studies on genome wide changes in cartilage and subchondral bone. Rushton et al present elegant work characterizing the genome-wide DNA methylation profile in articular cartilage from normal and OA-affected hip and OA-affected human knee joints. Results show that normal and OA hip cartilage have a unique methylation profiles, with 5322 differentially methylated loci (DMLs) identified between both groups. They also show that OA hip and knee samples can be separated into two clusters based on their methylation profile. In total 15239 DMLs were identified between the two hip OA clusters, with an enrichment of genes involved in inflammation and immunity. The identification and isolation of subgroups with different methylation profiles may also be critical for the development of effective disease prevention and treatment, and to characterize the different OA phenotypes such as inflammatory, aging and metabolicrelated.
\end{abstract}




\section{Editorial}

Osteoarthritis (OA) pathogenesis involves cartilage degradation, synovial inflammation, subchondral bone sclerosis, degeneration of ligaments and meniscus, and hypertrophy of the joint capsule. There can also be changes in periarticular muscles, nerves, bursa, and local fat pads that may contribute to OA. The manifestation of pathological changes in all joint tissues define $O A$ as a disease of the 'whole' joint, as an organ, resulting in organ dysfunction or joint failure (1). OA is a multifactorial disease in which gender, age, obesity and environment play a main role. OA has also a strong genetic component (2), however numerous studies failed to replicate genes, at GWAS significance level $\left(5.10^{-8}\right)$, with a full susceptibility to the disease. It has been proposed that this could be due to low penetrance polymorphisms in the population. Alternatively, it has been also proposed that this could, at least in part, be accounted for by inheritance of epigenetic modifications (3).

Epigenetics is defined as heritable changes in gene expression without changes in the DNA sequence. The term "heritable" refers to changes mainly between cell divisions and, sometimes, between generations; though in mammals the transgenerational inheritance is limited because germ cells, which give rise to the gametes, reset their epigenome prior to their reprogramming to a totipotent state (4). There are three main mechanisms involved in epigenetic regulation: i) posttranscriptional modification of histones, that alters chromatin conformation and thus the accessibility to the transcriptional machinery; ii) non-coding RNAs (i.e. microRNAs), acting post-transcriptionally in the regulation of gene expression through binding to target mRNAs, and iii) DNA methylation. DNA methylation is the best characterized epigenetic mechanism and consists of the addition of a methyl group $\left(\mathrm{CH}_{3}\right)$ from the methyl donor S-adenosylmethionine (SAM) to a cytosine that is $5^{\prime}$ to guanine (CpG sites) to form methylated cytosine $(5 \mathrm{mC})$. This reaction is catalyzed by a family of enzymes called DNA methyltransferases (DNMTs). The major outcome of DNA methylation is gene silencing when this process takes place in high $\mathrm{CpG}$ density 
regions of promoters (termed $\mathrm{CpG}$ islands); in contrast, when methylation occurs in gene bodies it leads to increased gene expression, probably to prevent incorrect transcriptional initiation (5). Most tissue-specific methylation occurs at regions of lower CpG density close to $\mathrm{CpG}$ islands (termed $\mathrm{CpG}$ shores) and correlates with transcriptional repression of the corresponding gene (3).

It has been proposed that epigenetics is involved in the phenotypic modulation that articular chondrocytes undergo during OA, generating these "altered" chondrocytes that overexpress cartilage-degrading proteases (collagenases and aggrecanases) and inflammatory mediators, therefore breaking the homeostatic balance toward extracellular matrix (ECM) degradation and probably playing a decisive role in the progression of the disease $(6,7)$. In this context, there have been several candidate-gene based studies investigating the role of DNA methylation in OA including growth differentiation factor-5 (GDF-5), manganese superoxide dismutase (MnSOD), interleukin-1 beta (IL-1 $\beta$ ) and several matrix-degrading proteinases (MMP-3, MMP-9, MMP-13, MMP-14 and ADAMTS-4). Moreover, a work by de Andrés et al (8) demonstrated a novel mechanism for inflammatory gene regulation in $O A$ chondrocytes, the demethylation of an enhancer that allows the binding of NF-K $\beta$ to facilitate the transcription of inducible nitric oxide synthase (iNOS).

All of these prior studies on DNA methylation focused on a single or a small number of genes. In this issue of Arthritis \& Rheumatology, Rushton et al present elegant work characterizing the genome-wide DNA methylation profile of normal and hip cartilage and knee cartilage (9). This study shows that normal and OA hip cartilage have a unique methylation profile, with 5322 differentially methylated loci (DMLs) identified between both groups. They also show that OA hip samples can be separated into two clusters based on their methylation profile. In total 15239 DMLs were identified between the two clusters, with an enrichment of genes involved in inflammation and immunity. These results confirm previous findings by our group in which two clusters of 
knee OA patients, with enrichment of similar genes involved in inflammation and immunity, had also been described (10). These two studies support the existence of an inflammatory OA phenotype and strengthen the consideration that, at least, part of the pathogenesis of OA can be attributable to epigenetics and that analysis of DNA methylomes may help to understand the complex molecular basis of this heterogeneous disease.

The study published in this issue of Arthritis \& Rheumatology also shows that cartilage DNA methylome is able to distinguish joint-specific OA. A comparison between hip OA and knee OA revealed 5547 DMLs between these two groups, including DMLs in several genes known to be involved in OA pathogenesis such as ADAM12, ADAMTS5, CHST11, GDF5 or MCF2L. These findings confirm that hip and knee OA are two diseases that share some common pathogenesis pathways but also with clear differences between them.

The role played by DNA methylation in OA is beginning to be investigated and the study by Rushton et al makes an important contribution in this respect. However, further efforts should be directed to address additional important and unresolved issues. The DNA methylome of other OA joint tissues such as synovial membrane and subchondral bone needs to be characterized. Information in this regard is limited to a recent genome-wide DNA methylation study which showed differential methylated regions between osteoporotic and $\mathrm{OA}$ bone (11). Interestingly, these regions were enriched in genes associated with cell differentiation and skeletal embryogenesis, such as those in the homeobox superfamily. These results are very interesting because similar results involving the homeobox superfamily were found in both genome-wide DNA methylation studies performed in OA cartilage $(9,10)$. Furthermore, joint morphology is a very relevant risk factor for OA development and progression. In-depth analysis of these results could help understand the connection between joint morphology and OA. 
Another important point to investigate is whether and how inflammatory stimuli are able to induce changes in the methylation pattern of OA (and vice-versa). In this sense, many proteinases are highly induced by inflammatory cues such as cytokines; in line with this, the two main pro-inflammatory cytokines involved in $O A, I L-1 \beta$ and TNF- $\alpha$, are able to induce changes in the methylation pattern of specific genes (12). A very recent study suggested a role for 5 -hydroxymethylcytosines $(5 \mathrm{hmC})$ in $\mathrm{OA}$ as inflammatory cytokines (IL-1 $\beta$, TNF- $\alpha$ and Oncostatin M) modulate the expression of TET1 (one of the enzymes that converts $5 \mathrm{mC}$ to $5 \mathrm{hmC}$ ) in OA chondrocytes. Increased TET expression led to an enrichment of $5 \mathrm{hmC}$ in $\mathrm{CpG}$ sites of gene bodies and promoters of some OA-related genes such as MMP-1, -3 and -13 , thereby increasing their expression in $\mathrm{OA}(13)$.

Epigenetic factors, including DNA methylation, histone modifications, and microRNAs may play central roles in controlling changes in gene expression and genomic instability during aging (14); aging and age-related diseases are generally characterized on the one hand by genome-wide hypomethylation, leading to genomic instability and inefficient gene repression, and on the other hand by promoter-specific hypermethylation, that leads to loss of expression control and inappropriate silencing $(15,16)$. The understanding of the specific mechanisms as well as the different pathways involved in the epigenetic modifications underlying aging and/or $O A$ is another major focus of research that it is necessary to explore.

DNA methylation and mitochondrial dysfunction is another interesting topic. Mitochondria play a role in the pathogenesis of $O A(17)$ and mitochondria and the mitochondrial genome modulate the nuclear epigenome (18). In line with this, carriers of the mitochondrial DNA (mtDNA) haplogroup $\mathrm{J}$ show increased levels of global DNA methylation when compared with other mtDNA variants (19). Consistently, cybrids from this haplogroup also show higher methylation levels than cybrids containing other mtDNA haplogroups (19); carriers of this haplogroup also show different DNA methylation patterns than carriers of the most common haplogroup $\mathrm{H}$, mainly in genes 
related to inflammation and apoptosis (personal data). Further, Kelly et al found that mouse embryonic stem cells containing different mtDNA haplogroups on a uniform nuclear background were associated with different gene expression and DNA methylation profiles (20). Conceivably, mitochondrial reactive oxygen species (ROS) production, which differs among the mtDNA haplogroups (21), represents an important retrograde signaling stimulus for nuclear epigenomic DNA modifications (22). The emergence of the mitochondrial genome as one of the regulators of the nuclear DNA function is a very important issue and should also be studied in detail.

Metabolic alterations such as type 2 diabetes mellitus and obesity are consistently associated with OA suggesting the existence of a metabolic phenotype of OA (23). Moreover, there is evidence that obese and diabetic patients show different epigenetic marks than non-obese and non-diabetic individuals (24). Altogether, these data indicate that metabolic alterations modify the DNA methylome suggesting an interesting connection between methylation and the newly defined metabolic phenotype of $O A$.

Dietary changes are also able to induce changes on the DNA methylome since SAM, the main methyl donor, is acquired through the intake of folic acid and vitamins $\mathrm{B} 6$ and $\mathrm{B} 12$, therefore, a decrease in the uptake of these dietary supplements could lead to a global hypomethylation status. In a recent article, Barua et al demonstrated that the offspring of pregnant mice fed a diet high in folic acid exhibit significant changes in DNA methylation patterns of their neuronal tissue (25). In the context of OA, some studies even propose the use of SAM as an effective complementary medicine (26), although its effectiveness has not been conclusively proven. Improved clinical trials must be carried out regarding to the intake of methyl donors and also to explore its potential mechanisms of action such as changes in DNA methylation and associated reduction of inflammatory mediators and cartilage-degrading proteinases.

Methylation is a dynamic process prone to changes in the environment. For this reason, in-depth studies are necessary to determine if the methylation changes that 
take place in $\mathrm{OA}$ are cause or consequence of the changes produced by the disease itself, or even a combination of both scenarios. In this sense, studies involving the analysis of DNA methylation changes in in vitro models of chondrogenesis could be useful. Chondrogenesis has been revealed as a process involved in OA pathogenesis and it has been proposed that defective chondrogenesis could be responsible for defects in cartilage repair contributing to OA (27). Work by Ezura et al (28) described low DNA methylation levels of CpG-rich promoters of genes regulating chondrogenesis, including SOX9 or RUNX2, in human synovium-derived mesenchimal stem cells. Interestingly, low methylation levels in CpG promoter for RUNX2 in OA chondrocytes were also detected in both studies by Rushton et al (9) and FernándezTajes et al (10). Together, these data indicate that changes in the methylation patterns of cells with chondrogenic capacity occur and should also be the focus of future studies.

In conclusion, the study by Rushton et al strengthens the role of DNA methylation in $\mathrm{OA}$ and makes a call to research community to increase the efforts on the role played by epigenetics in the development of $O A$. In regard to therapeutic implications, the characterization and analysis of cartilage DNA methylomes offers not only potential therapeutic targets for the treatment of $\mathrm{OA}$, but also enables the design of a map of epigenetic marks that can help develop potential biomarkers for diagnosis, prognosis, drug-response, chondrogenesis or homeostasis from high-resolution screening technologies, as proposed for other diseases (29). The identification and isolation of subgroups with different methylation profiles may also be critical for the development of effective treatment and disease prevention. Answering the question in the title of this editorial, it is time for epigenetics in $\mathrm{OA}$, time to elucidate the role of epigenetics to characterize the different $O A$ phenotypes (Figure 1) such as inflammatory, aging and metabolic-related. 
Acknowledgements. We are grateful to Martin Lotz MD, from the Scripps Research Institute (CA), for his suggestions and comments.

Funding. This study was supported by grants from Fondo Investigacion Sanitaria PI 12/0329, with a contribution of funds from FEDER (European Community). Ignacio Rego-Pérez is supported by Contrato Miguel Servet-Fondo Investigacion Sanitaria (CP12/03192).

Contributors. FJB and IRP have written and approved the final version of this editorial.

Competing interests. None 


\section{REFERENCES}

1. Blanco FJ. Osteoarthritis: something is moving. Reumatol Clin. 2014;10(1):4-5.

2. Valdes AM, Spector TD. Genetic epidemiology of hip and knee osteoarthritis. Nat Rev Rheumatol. United States; 2011. p. 23-32.

3. Barter MJ, Bui C, Young DA. Epigenetic mechanisms in cartilage and osteoarthritis: DNA methylation, histone modifications and microRNAs. Osteoarthritis Cartilage. 2012;20(5):339-49.

4. Feng S, Jacobsen SE, Reik W. Epigenetic reprogramming in plant and animal development. Science. 2010;330(6004):622-7.

5. Hellman A, Chess A. Gene body-specific methylation on the active $X$ chromosome. Science. 2007;315(5815):1141-3.

6. Roach HI, Yamada N, Cheung KS, Tilley S, Clarke NM, Oreffo RO, et al. Association between the abnormal expression of matrix-degrading enzymes by human osteoarthritic chondrocytes and demethylation of specific CpG sites in the promoter regions. Arthritis Rheum. 2005;52(10):3110-24.

7. Reynard LN, Loughlin J. Genetics and epigenetics of osteoarthritis. Maturitas. 2012;71(3):200-4.

8. de Andrés MC, Imagawa K, Hashimoto K, Gonzalez A, Roach HI, Goldring MB, et al. Loss of methylation in CpG sites in the NF-KB enhancer elements of inducible nitric oxide synthase is responsible for gene induction in human articular chondrocytes. Arthritis Rheum. 2013;65(3):732-42.

9. Rushton M, Reynard L, Barter M, Refaie R, Rankin K, Young D, et al. Characterization of the cartilage DNA methylome in knee and hip osteoarthritis. Arthritis \& Rheumatology. 2014.

10. Fernandez-Tajes J, Soto-Hermida A, Vazquez-Mosquera ME, Cortes-Pereira E, Mosquera A, Fernandez-Moreno M, et al. Genome-wide DNA methylation analysis of articular chondrocytes reveals a cluster of osteoarthritic patients. Ann Rheum Dis; 2013.

11. Delgado-Calle J, Fernández AF, Sainz J, Zarrabeitia MT, Sañudo C, García-Renedo R, et al. Genome-wide profiling of bone reveals differentially methylated regions in osteoporosis and osteoarthritis. Arthritis Rheum. 2013;65(1):197-205.

12. Hashimoto K, Oreffo RO, Gibson MB, Goldring MB, Roach HI. DNA demethylation at specific CpG sites in the IL1B promoter in response to inflammatory cytokines in human articular chondrocytes. Arthritis Rheum. 2009;60(11):3303-13.

13. Taylor SE, Smeriglio P, Dhulipala L, Rath M, Bhutani N. A global increase in 5hydroxymethylcytosine levels marks osteoarthritic chondrocytes. Arthritis Rheumatol. 2014;66(1):90-100.

14. Berdasco M, Esteller M. Hot topics in epigenetic mechanisms of aging: 2011. Aging Cell. 2012;11(2):181-6.

15. Portela A, Esteller M. Epigenetic modifications and human disease. Nat Biotechnol. 2010;28(10):1057-68.

16. Johnson AA, Akman K, Calimport SR, Wuttke D, Stolzing A, de Magalhães JP. The role of DNA methylation in aging, rejuvenation, and age-related disease. Rejuvenation Res. 2012;15(5):483-94.

17. Blanco FJ, Rego I, Ruiz-Romero C. The role of mitochondria in osteoarthritis. Nature Reviews Rheumatology. 2011;7(3):161-9.

18. Horan MP, Cooper DN. The emergence of the mitochondrial genome as a partial regulator of nuclear function is providing new insights into the genetic mechanisms underlying agerelated complex disease. Hum Genet. 2014;133(4):435-58.

19. Bellizzi D, D'Aquila P, Giordano M, Montesanto A, Passarino G. Global DNA methylation levels are modulated by mitochondrial DNA variants. Epigenomics. 2012;4(1):17-27. 
20. Kelly RD, Rodda AE, Dickinson A, Mahmud A, Nefzger CM, Lee W, et al. Mitochondrial DNA haplotypes define gene expression patterns in pluripotent and differentiating embryonic stem cells. Stem Cells. 2013;31(4):703-16.

21. Kenney MC, Chwa M, Atilano SR, Falatoonzadeh P, Ramirez C, Malik D, et al. Molecular and bioenergetic differences between cells with African versus European inherited mitochondrial DNA haplogroups: Implications for population susceptibility to diseases. Biochim Biophys Acta. 2014;1842(2):208-19.

22. Schroeder EA, Raimundo N, Shadel GS. Epigenetic silencing mediates mitochondria stress-induced longevity. Cell Metab. 2013;17(6):954-64.

23. Sowers M, Karvonen-Gutierrez CA, Palmieri-Smith R, Jacobson JA, Jiang $Y$, Ashton-Miller JA. Knee osteoarthritis in obese women with cardiometabolic clustering. Arthritis Rheum. 2009;61(10):1328-36.

24. Martínez JA, Milagro FI, Claycombe KJ, Schalinske KL. Epigenetics in adipose tissue, obesity, weight loss, and diabetes. Adv Nutr. 2014;5(1):71-81.

25. Barua S, Kuizon S, Chadman KK, Flory MJ, Brown WT, Junaid MA. Single-base resolution of mouse offspring brain methylome reveals epigenome modifications caused by gestational folic acid. Epigenetics Chromatin. 2014;7(1):3.

26. De Silva V, El-Metwally A, Ernst E, Lewith G, Macfarlane GJ, Medicines ARUWGoCaA. Evidence for the efficacy of complementary and alternative medicines in the management of osteoarthritis: a systematic review. Rheumatology (Oxford). 2011;50(5):911-20.

27. Blanco FJ, Ruiz-Romero C. New targets for disease modifying osteoarthritis drugs: chondrogenesis and Runx1. Ann Rheum Dis. 2013;72(5):631-4.

28. Ezura Y, Sekiya I, Koga H, Muneta T, Noda M. Methylation status of CpG islands in the promoter regions of signature genes during chondrogenesis of human synovium-derived mesenchymal stem cells. Arthritis Rheum. 2009;60(5):1416-26.

29. Heyn $\mathrm{H}$, Esteller M. DNA methylation profiling in the clinic: applications and challenges. Nat Rev Genet. 2012;13(10):679-92. 